\title{
Editorial
}

\section{Intelligent Connected Vehicle as the New Carrier Towards the Era of Connected World}

\author{
ZHUANG Hanyang ${ }^{\mathrm{a}}$ (庄瀚洋), QIAN Yeqiang ${ }^{\mathrm{a}}$ (钱烨强), YANG Ming ${ }^{\mathrm{a}, \mathrm{b} *}$ (杨 明) \\ (a. University of Michigan - Shanghai Jiao Tong University Joint Institute; b. Department of Automation, \\ Shanghai Jiao Tong University, Shanghai 200240, China)
}

(C) Shanghai Jiao Tong University and Springer-Verlag GmbH Germany, part of Springer Nature 2021

Human beings have been kept pursuing of higher efficiency and better safety to move people and things around since thousands of years ago. In modern society, vehicles are therefore invented and utilized to boost the speed and enhance the safety. In recent years, rapid development of information technology has brought human into a new era of connected world. Internet and smartphones have made it extremely easy to get access to anyone from anywhere any time. In this background, intelligent connected vehicles (ICVs) have been proposed and investigated. In the similar manner as the smartphones, ICVs are expected to be the next generation carrier for people to get connected to the world.

ICVs are equipped with novel sensors, controllers, and actuators to understand the environment, make decisions, and take actions, respectively. The word "intelligent" indicates that the vehicle should be able to handle unexpected events on the road. The word "connected" means that the information of each vehicle should be shared and considered globally. Full autonomy and full connection are the ultimate goals of ICV industry. Unfortunately, we are still far away from this goal; therefore, continuous efforts shall be made to step further to this destination.

As the ICV consists of multiple subsystems and is across different disciplines, the overall improvement requires the innovation in each aspect. Under this circumstance, the Special Issue on Intelligent Connected Vehicle at Journal of Shanghai Jiao Tong University (Science) has been organized to broaden the perspective, promote the interdisciplinary collaboration, and report the state-of-the-art works.

Thanks to the active participations of the authors from leading universities and companies, twenty papers have been recognized qualified and they are published in this special issue. As mentioned above, ICV is a multi-

Received: 2021-03-25 Accepted: 2021-03-26

$*$ E-mail: mingyang@sjtu.edu.cn disciplinary subject; the published papers also cover a wide range of research topics from the advanced driving assistance systems to high-level autonomous driving systems. In detail, these papers present cutting edge results in perception, mapping, control, planning, simulation, vehicle-to-everything (V2X), etc. A brief introduction is given as follows.

The perception system is the key of the ICV which provides accurate and reliable detection results for decision making and planning. The first paper entitled "Camera-Radar Fusion Sensing System Based on Multi-Layer Perceptron" presents a camera-radar fusion system to enhance the perception result by building a multi-layer perceptron structure.

The second paper "Wavelet Transform-Based HighDefinition Map Construction From a Panoramic Camera" aims at constructing the high-definition map by panoramic camera. The wavelet-transform is applied to generate sharp and consistence base images for highdefinition map annotation.

The next paper named "Lightweight Method for Vehicle Re-identification Using Reranking Algorithm Based on the Topology Information of Surveillance Network" focuses on reducing the weight and data annotation requirement for the task of vehicle re-identification. The work serves the cooperative infrastructure to track the vehicles in a better way.

Digital twin is built by digitalizing the real world and reconstructing it in simulation environment. The paper "Intelligent Analysis of Abnormal Vehicle Behavior Based on Digital Twin" presents a novel framework to generate digital twin, and the experiments demonstrate that this framework performs well in detecting a range of abnormal behaviors.

"Efficient Online Vehicle Tracking for Real-Virtual Mapping Systems", the fifth paper, combines nonrecursive temporal windows search with delayed output and produces stable trajectories despite noisy detection responses. The framework is efficient and flexible for 
online vehicle tracking-by-detection task.

The next paper entitled "Multi-Object Tracking Strategy of Autonomous Vehicle Using Modified Unscented Kalman Filter and Reference Point Switching" aims at modifying the unscented Kalman filter for multiple-object tracking task. A reference point switching procedure is introduced to improve accuracy and consistency.

Remote control is an important backup for ICV to increase the system redundancy. The design of shared control method can utilize both the vehicle intelligence and human experience. The paper "Intelligent-Assist Algorithm for Remote Shared-Control Driving Based on Game Theory" presents a haptic shared-control algorithm, in which the man-machine interaction is modeled according to game theory so that the system automatically places extra emphasis on driving safety or driver's intention in different situations.

Vehicle control is the fundamental for ICV which is the topic of the following three papers. Lane keeping is the basis of vehicle control, but strongly related to the vehicle safety and passenger comfort. The eighth paper "Stochastic Model Predictive Control Approach to Autonomous Vehicle Lane Keeping" presents a stochastic model predictive control method to reach better effectiveness and smaller steady-state error.

Cooperative adaptive cruise control is studied by the next paper named "Cooperative Adaptive Cruise Control Using Delay-Based Spacing Policy: A Robust Adaptive Non-Singular Terminal Sliding Mode Approach". The delay-based spacing policy is adopted and a seven-vehicle platoon is simulated to verify the algorithm.

The next paper "Parameter Identification of Magic Formula Tire Model Based on Fibonacci Tree Optimization Algorithm" focuses on improving the vehicle tire model parameter identification. The results are beneficial for vehicle dynamic modeling which may improve the control stability and precision.

High-precision map is the key component for ICV. Also, it is important for national security. Therefore, how to develop high-precision map in China is an interesting and challenging topic which is carried out by the eleventh paper with title of "Developing High-Precision Maps for Automated Driving in China: Legal Obstacles and the Way to Overcome Them".

The next paper focuses on V2X application. Mobile edge computing (MEC) server is the roadside equipment to provide additional information to ICV. The paper "Service Caching and Task Offloading for MECenabled Intelligent Connected Vehicles" aims at determining the optimal service caching and task offloading decisions to minimize the overall system delay.

The vehicle-to-vehicle $(\mathrm{V} 2 \mathrm{~V})$ connection provides the capability of multi-agent formation. The next paper named "Obstacle Avoidance of Multi-Agent Formation Process Based on Deep Reinforcement Learning" presents an improved DQN algorithm to realize the multi-agent formation process in the desired obstacle avoidance and collision avoidance.

The paper entitled "IoT System for Intelligent Firefighting in the Electric Power Industry" aims at proposing a smart management system to prevent fire. The Internet of things technology is used to perform multiparty coordination.

The fifteenth paper "Curvature Adaptive Control Based Path Following for Automatic Driving Vehicles in Private Area" demonstrates a curvature adaptive control method to handle the vehicle steering action in irregular private areas. The proposed method reaches higher accuracy with better resistance to the curvature fluctuation.

Evaluation system is essential to validate the safety and reliability of ICV. In the paper "Integrated Framework for Test and Evaluation of Autonomous Vehicles", a scientific and complete framework is proposed which includes the design of test scenarios, the selection of evaluation indexes, and the establishment of the evaluation system.

A two-wheel self-balancing vehicle is a very basic system to develop fundamental system control algorithms. The seventeenth paper "Control System of Two-Wheel Self-Balancing Vehicle" aims at improving the cascade coupling control method of two-wheel vehicle to reach smoother and faster movement.

Path planning helps ICV to find the optimal sequence of way points to drive. The next paper "Real-Time Trajectory Planning for On-road Autonomous TractorTrailer Vehicles" focuses on developing the planning algorithm for a tractor-trailer vehicle. The complex vehicle kinematics is modeled and a framework is proposed to generate the high-quality, obstacle-free trajectory.

The paper entitled "Collision-Free Path Planning with Kinematic Constraints in Urban Scenarios" also focuses on the path planning topic. It proposes a method combining sampling-based method and optimization-based method to generate a collision free path with continuous curvature in urban scenarios.

The last paper named "Iterative-Reweighting-Based Robust Iterative-Closest-Point Method" solves the conventional point cloud registration problem with a higher efficiency and consistency. The penalty function based robust point-to-point iterative closest point problem is formulated to a uniform constrained nonlinear optimization problem and solved by an iterative reweighting-based method.

We are grateful for the contributions offered by the authors, reviewers, editors, as well as the great supports from Innovation Center of Intelligent Connected Vehicle at Shanghai Jiao Tong University. All their passions and specialties in the ICV technology make the success of this special issue. It is our hope to see that this special issue will make impacts on inspiring more original and innovative research works. 